\title{
Estimation of Functional Independence Measure Motor Score Based on a Trunk Control Model
}

\author{
Taku HIROTA*, Yuri HAMADA*, Takashi KABURAGI** and Yosuke KURIHARA* \\ * Aoyama Gakuin University, 5-10-1 Fuchinobe, Chuo-ku, Sagamihara, Kanagawa 252-5258, Japan \\ ** International Christian University, 3-10-2 Osawa, Mitaka-shi, Tokyo 181-8585, Japan
}

\begin{abstract}
The functional independence measure (FIM) is a metric of activities of daily living in stroke patients, but its accurate assessment requires experienced nurses. Therefore, we propose a system for estimating FIM scores easily at home based on angular velocities measured from specific bodily movements, performed only once, using gyro sensors. Here, we focus on the FIM motor score $(\mathrm{mFIM})$ and determine whether "rising from a bed" or the "sit-to-stand movement" is the optimal specific bodily movement. We constructed a model considering the differences in convergence speeds between the movements of healthy people and those of stroke patients. We estimated the mFIM by performing regression analysis with the parameters utilized in the developed model as explanatory variables and mFIMs assessed by experienced nurses as objective variables. In our experiments, the mean absolute error of the angular velocity in the spine direction for rising from a bed was the smallest at 9.53.
\end{abstract}

Keywords: FIM, Trunk function, Angular velocity

\section{INTRODUCTION}

In fiscal year $2019,28.4 \%$ of the total population in Japan was aged 65 years or older. By 2060, it is estimated that this demographic will account for $40 \%$ of the total population [1]. As a result of this rapid aging, approximately 1.17 million patients have become bedridden and are forced to lead crippled lives with residual symptoms such as motor paralysis and gait disorders caused by stroke [2]. Stroke is the second leading cause of long-term care requirements [3]. Immobile body function declines in stroke patients based on the weakening of limb muscles, stiffening of joints, and loss of strength. Therefore, appropriate rehabilitation at an early stage is essential. Although rehabilitation is necessary for recovering declining functions, patients may experience a decline in motivation during the rehabilitation process because they must continuously perform painful movements. During rehabilitation, it is critical for patients to confirm the recovery process of declining functions objectively to increase motivation.

The functional independence measure (FIM), which is a metric of activities of daily living (ADL) in stroke patients, is one of the most reliable physical therapy assessments that can be utilized objectively for the recovery process during rehabilitation. The FIM measures two unidimensional domains of motor function (13 items) and cognitive function (five items). Each item is rated on a scale from one (total dependence) to seven (total independence) [4-7]. Hence, the FIM cognitive score and FIM motor score (mFIM), which are the sums of the scores for each item in their respective categories, are in the range of 5-35 and 13-91, respectively. However, because FIM assessment can only be performed by experienced and highly trained nurses, and it is difficult to evaluate FIM scores accurately for patients who are engaged in rehabilitation in a home environment on a daily basis. If patients can easily assess their FIM scores at home, they can closely monitor their ADL recovery status to encourage further rehabilitation.

In a related study on estimating the FIM motor score (mFIM), a method for predicting the mFIM at discharge based on age, duration from stroke onset to admission, mFIM on admission, total score of FIM cognitive items on admission, sex, and type of stroke was proposed [8]. Additionally, we proposed an mFIM estimation method for measuring a patient's bodily movements during "rising from a bed" and "sit-to-stand (STS) movement" utilizing gyro sensors attached to the patient's sternum [9]. In [9], we focused solely on the mFIM because the FIM cognitive score should be evaluated while communicating with patients. Therefore, considering movement instability caused by insufficient balance control, we developed a system to estimate total scores for 13 FIM motor items based on the target body movements.

In this study, we aim to construct a system that enables stroke patients to easily estimate their current mFIM by performing a specific bodily movement only once in a home environment; we also determine whether 


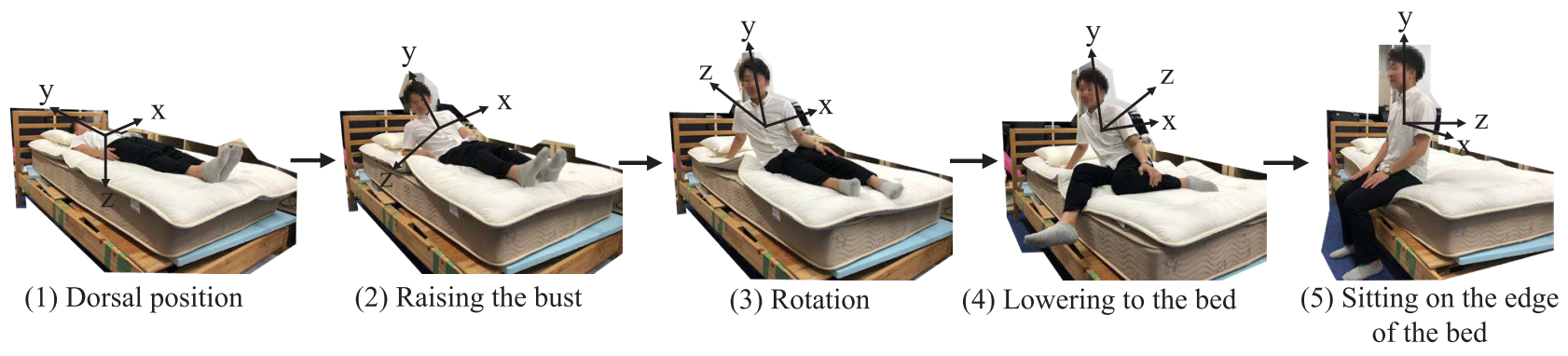

Figure 1: Phases of rising from a bed

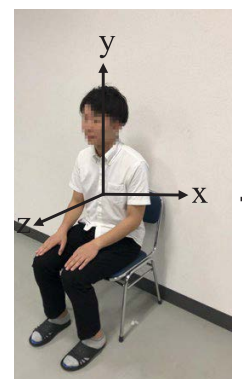

(1) Movement begins

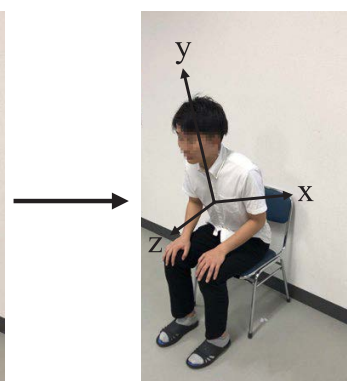

(2) Transfer

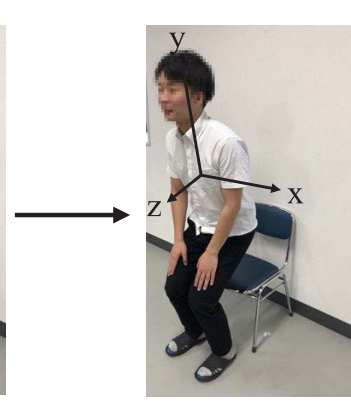

(3) Extension

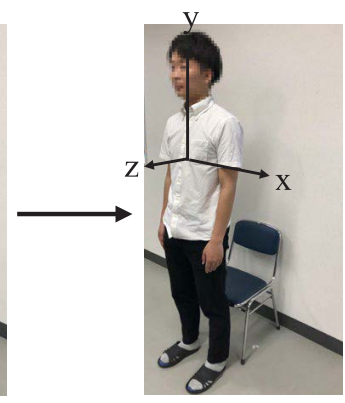

(4) Stabilization

Figure 2: Phases of STS movement

rising from a bed or the STS movement is the optimal movement. In the proposed system, we constructed a model that considers how fast a patient's bodily movements converge to the target position. The mFIM can be estimated by performing Gaussian process regression analysis with the parameters utilized in the constructed model as explanatory variables and the mFIMs assessed by highly trained nurses as objective variables.

\section{TARGET BODILY MOVEMENTS}

In this paper, we propose a method for estimating FIM scores by measuring the movements of a patient performing specific movements. As specific movements, rising from a bed and STS movement, which are the preliminary steps for standing balance and walking, are targeted because these movements are essential for preventing patients from becoming bedridden. Additionally, the importance of trunk assessment and intervention was described in [10] because trunk performance decreases following a stroke and trunk performance is strongly related to assessments of balance and functional activity. Therefore, we focused on patient trunk movements during the target movements.

\subsection{Rising from a bed}

Figure 1 presents the sequence of movements performed during rising from a bed. The movements are categorized into five phases as follows:
- Phase 1: Dorsal position

- Phase 2: Raising the bust

- Phase 3: Rotation

- Phase 4: Lowering to the bed

- Phase 5: Sitting on the edge of the bed

To analyze trunk movement during the target movements, we defined a relative Cartesian coordinate system with the center of the human waist as the origin, $\mathrm{x}$ axis running from the right femur to the left femur (horizontal plane), $y$ axis in the direction of the spinal cord (frontal plane), and $\mathrm{z}$ axis running from the front to the back (sagittal plane), as shown in Fig. 1. This coordinate system moves according to the movement of the trunk of the patient. As shown in Fig. 1, rotational movement is the main type of movement during the target bodily movement. Patients with severe strokes and low mFIMs have greater difficulty in controlling their body to the target position compared to patients with high mFIMs based on insufficient balance control and muscle strength. Therefore, it takes more time for them to get up by twisting their trunk and rocking from side to side. In contrast, because mildly symptomatic patients with high mFIMs have adequate physical functions, the series of movements can be accomplished smoothly and quickly.

\subsection{STS movement}

Figure 2 presents the sequence of STS movement and corresponding relative Cartesian coordinate system. The ability to perform STS movement is strongly influenced 
by the height of the chair seat, use of armrests, and foot position [11]. STS failures for elderly people and those with mobility impairment can result from weakness, poor balance control, and coordination impairment, resulting in insufficient energetic effort [12]. The STS movement was divided into the following phases [13]:

- Phase 1: Movement begins

- Phase 2: Transfer

- Phase 3: Extension

- Phase 4: Stabilization

As shown in Fig. 2, we defined a relative Cartesian coordinate system with the patient's waist as the origin, $\mathrm{x}$ axis running from the right femur to the left femur, $y$ axis in the direction of the spine, and $\mathrm{z}$ axis pointing toward the front. In this movement, the trunk is bent forward about the hip joint and then bent backward about the hip joint in a rotational movement. In general, hemiplegic patients with lower mFIMs experience difficulty in hip flexion during phase 2. For this reason, similar to the case of rising from a bed, they attempt to stand up by twisting their trunk or rocking from side to side, but the forward shift of their weight is insufficient, making it difficult for them to stand up smoothly. In contrast, it has been reported that healthy people with higher mFIMs stand up quickly and demonstrate a small amount of trunk lateral flexion, trunk lateral shift, and trunk rotation, despite large amounts of trunk forward flexion and upward activity during the STS movement [14]. Therefore, when evaluating STS movements, the forward-backward tilt (x axis), sway (y axis), and left-right sway of the trunk ( $\mathrm{z}$ axis) are important measures.

\section{PROPOSED METHOD}

\subsection{Trunk control model based on damped oscillation}

We propose a model that is accompanied by trunk functions. As mentioned in Section 2, because patients with low mFIMs (stroke patients) have insufficient trunk performance, their movements are unstable and require more time to complete. In contrast, the movements of individuals with high mFIMs (healthy people) are completed smoothly and quickly based on their stable trunk performance. In this study, to analyze the rotational movements representing the movement characteristics of stroke patients and healthy people, we constructed the following model based on damped oscillation:

$$
f(k)=A e^{-\gamma k} \cos (\alpha k+\beta) H(k-T),
$$

Where $k(=1,2, \ldots, L)$ is the discrete time and $L$ is the length of the time series data. $A$ is the magnitude of the initial movement and $\gamma$ is the convergence of the movement. For a stroke patient's movement, the initial magnitude of the movement $(A)$ and convergence of the movement $(\gamma)$ are expected to be smaller based on insufficient trunk performance. Additionally, $\alpha$ is the oscillation frequency, which represents the stability of movement. The phase $\beta$ controls the timing of waves generated by the movement. $H(\cdot)$ is the Heaviside step function, which captures the time until the target bodily movements start by setting a parameter $T$. By determining the five parameters $A, \gamma, \alpha$, $\beta$, and $T$, the target bodily movements are characterized by the model $f(k)$. Because we focus on the rotational movements in the $\mathrm{x}, \mathrm{y}$, and $\mathrm{z}$ directions from each of the two target bodily movements, six models were constructed. By utilizing all five features $A, \gamma, \alpha, \beta$, and $T$ in each of these models, we can estimate the mFIMs for each of the $\mathrm{x}, \mathrm{y}$, and $\mathrm{z}$ axes for each movement; we can also estimate the mFIM by combining all three axes for each movement.

\subsection{Signal processing flow scheme for mFIM estimation}

In the proposed method, we utilized gyro sensors, which are compact and relatively inexpensive devices, to measure the angular velocities associated with the rotational motion of the trunk in the relative Cartesian coordinate system during the target bodily movements. The angular velocity of each axis measured while rising from the bed and during STS movement is defined as $\omega(k)$. A signal processing flow based on the trunk control model and the measured angular velocities was adopted to estimate the mFIM by using each axis or combining all three axes for each movement. This process, shown in Fig. 3, consisted of two phases: preparation and estimation.

1) Preparation phase:

We aimed to construct regression equations to estimate mFIMs in the preparation phase. The signal processing flow scheme for the preparation phase consists of three stages, namely preprocessing, feature extraction, and regression analysis, as shown in Fig. 3. In the preprocessing stage, we utilize the Kalman smoother for denoising the angular velocities $\omega(k)$ measured by the gyro sensors because the angular velocities obtained from gyro sensors contain white noise. The state and observation equations are defined as follows:

$$
\begin{aligned}
& {\left[\begin{array}{c}
\theta(k+1) \\
\omega(k+1)
\end{array}\right]=\left[\begin{array}{cc}
1 & \Delta t \\
0 & 1
\end{array}\right]\left[\begin{array}{c}
\theta(k) \\
\omega(k)
\end{array}\right]+\left[\begin{array}{c}
v_{\theta}(k) \\
v_{w}(k)
\end{array}\right],} \\
& y(k)=\left[\begin{array}{ll}
0 & C
\end{array}\right]\left[\begin{array}{c}
\theta(k) \\
\omega(k)
\end{array}\right]+w(k),
\end{aligned}
$$




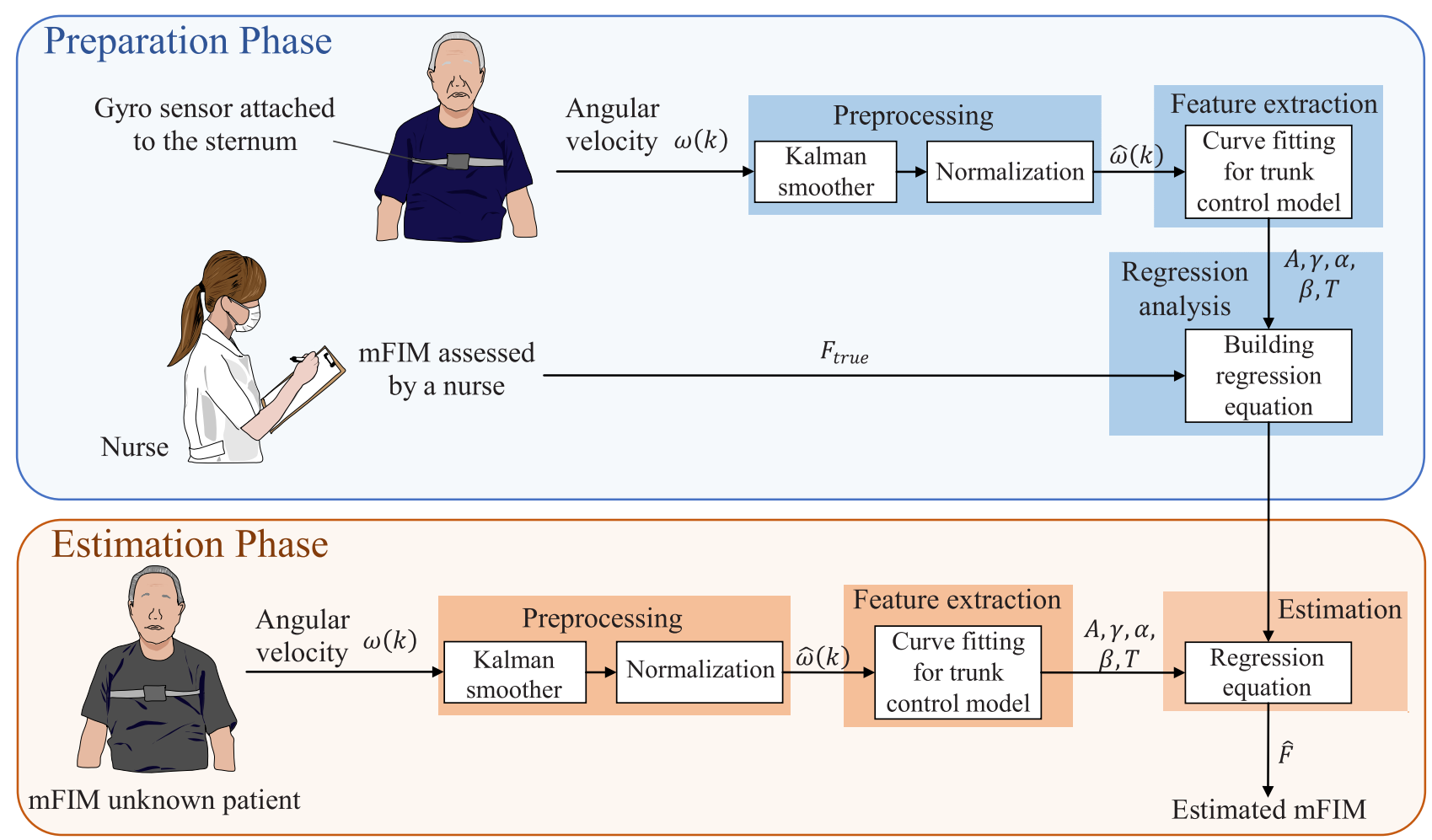

Figure 3: Signal processing flow scheme for estimating mFIMs

Where $\theta(k)$ represents angle, and the system noise and observation noise are denoted as $v(k)$ and $w(k)$, respectively. Additionally, $\Delta t$ and $C$ are the sampling interval and observation coefficient, respectively. After applying the Kalman smoother, we perform normalization with a mean of zero and standard deviation of one on the denoised angular velocities to reduce the time required to determine the five parameters of the model. Let $\hat{\omega}(k)$ be the angular velocity after preprocessing. To extract the features $A, \gamma, \alpha, \beta$, and $T$, of the trunk control model, curve fitting is performed to fit $\hat{\omega}(k)$ utilizing the model described in Eq. (1) in the feature extraction stage. For curve fitting, we utilized the interiorpoint method by solving the following minimization problem to minimize the squared error between the angular velocities $\hat{\omega}(k)$ after preprocessing and the output of the model $f(k)$ :

$$
\text { minimize }\{\hat{\omega}(k)-f(k)\}^{2} \text {. }
$$

By considering the features $A, \gamma, \alpha, \beta$, and $T$ as explanatory variables and the mFIMs $F_{\text {true }}$ assessed by highly trained nurses as objective variables, we obtained a regression equation for the regression analysis stage. The Gaussian process regression method was adopted to build the regression equation. In the case of combining all three axes, 15 features (the five features $A, \gamma, \alpha, \beta$, and $T$ for each axis) were used as explanatory variables.
2) Estimation phase:

The prediction phase of the signal processing flow scheme also consists of three stages: the preprocessing stage, feature extraction stage, and mFIM estimation stage, as shown in Fig. 3. In the preprocessing stage, similar to the previous section, we apply the Kalman smoother to the angular velocities $\omega(k)$ measured by gyro sensors on patients with unknown mFIMs. Subsequently, the denoised angular velocities were normalized and we obtained $\hat{\omega}(k)$. In the feature extraction stage, the interior point-method was applied to the angular velocities $\hat{\omega}(k)$ after preprocessing and we obtained the features $A, \gamma, \alpha, \beta$, and $T$. In the mFIM estimation stage, by inputting the features into the regression equation constructed in the preparation phase, we obtained the estimated mFIM $\hat{F}$. The above processing flow was applied to six $\omega(k)$ values measured around the $\mathrm{x}, \mathrm{y}$, and $\mathrm{z}$ axes for each target bodily movement, and the mFIM was also estimated in the case of combining all three axes for each target bodily movement.

\section{EXPERIMENTS FOR EVALUATING THE PROPOSED METHOD}

In this experiment, to verify the optimal combination of specific bodily movement and axis that is utilized for the 
regression equation, the mean absolute errors (MAEs) between the estimated mFIM $\hat{F}$ and $F_{\text {true }}$ assessed by the highly trained nurses were compared in the cases of applying each individual axis or combining all axes for both the rising from a bed and STS movement cases. Hence, eight MAEs were compared. Since the purpose of this study was to easily estimate the mFIM for patients in a home environment, we consider that the specific bodily movement for the proposed system must be simple so as not to burden the patient. Therefore, we did not apply cases combining multiple movements to estimate the mFIM in this experiment.

\subsection{Experimental system}

We utilized 3D gyro sensors as the basic elements of the measurement method and defined a coordinate system for measuring the angular velocity at each gyro sensor. If gyro sensors are used for a long duration, drift may occur. Drift can be corrected by subtracting the DC component measured in the stationary state until the start of the target bodily movements from the data for the entire measurement period. Under the conditions described above, we measured angular velocities during the target body movements. The gyro sensors used were Murata ENC-03J sensors, which are small and light with dimensions of $15.5 \times 8 \times 4.3$ and a weight of $2.7 \mathrm{~g}$. The three gyro sensors were arranged orthogonally and placed in a $50 \times 30 \times 20 \mathrm{~mm}$ case. The outputs of the gyro sensors should be positive when rotating in the directions of the arrows in Figs. 1 and 2. For measurement, the sensors were fixed to each participant's sternum utilizing a sports belt, which facilitated easy installation and adjustment for each participant.

\subsection{Experimental settings and participants}

We conducted experiments utilizing the method described above with the cooperation of 24 participants after obtaining their informed consent. The participants consisted of five healthy individuals and 19 patients $(62.7 \pm 9$ y old $)$ undergoing rehabilitation who had paralysis as a sequela of cerebrovascular diseases. To obtain the angular velocities $\omega(k)$, the $3 \mathrm{D}$ gyro sensors were attached to the sternum utilizing a sports belt and the participants were asked to perform the two target bodily movements. In this experiment, the participants who had paralysis performed the two target bodily movements without any support from a caregiver. The angular velocities $\omega(k)$ measured by the $3 \mathrm{D}$ gyro sensors were analog-to-digital converted at a sampling interval of $0.05 \mathrm{~s}$ $(\Delta t=0.05)$. Additionally, a different nurse for each patient (average work experience of $10.2 \pm 2.3 \mathrm{y}$ ) evaluated the mFIM.

For dataset collection, five healthy individuals and four patients were measured once each, and nine datasets were collected. Fifteen patients were measured multiple times at one-month intervals, and 35 datasets, including datasets with different mFIMs of the same patient due to rehabilitation-induced improvement, were obtained. As a result, we obtained a total of 44 datasets. One dataset was composed of the six $\omega(k)$ values and $F_{\text {true }}$. All experiments were conducted after receiving an ethical review and approval from Aoyama Gakuin University (permission no. H21-004). Informed consent was obtained before initiation of the experiment.

\subsection{Parameter settings}

When utilizing the Kalman smoother, the covariance matrix of the system noise $v(k)$ is $\left[\begin{array}{cc}50 & 0 \\ 0 & 50\end{array}\right]$ and the variance of the observation noise $w(k)$ is $5 \times 10^{-6}$. Additionally, the initial conditions of the state vector and posteriori error covariance matrix were set to a zero vector and identity matrix, respectively. Furthermore, $C$ was set to $0.1 \times 10^{-3}$ because the gyro sensors output a voltage of $0.1 \mathrm{mV}$ for an angular velocity of $1 \mathrm{rad} / \mathrm{s}$.

\subsection{Evaluation method}

The purpose of the proposed method is to estimate current mFIM using only one measurement trial of a patient's bodily movement in a home environment. Among the 44 datasets, even if there were multiple datasets from the same patient, the datasets were measured at least at 1-month intervals. Therefore, in this experiment, each dataset was regarded as an independent measurement of the patient's target bodily movement, and leave-one-dataset-out cross-validation was performed to verify the accuracy of current mFIM estimation based on one measurement trial. In other words, we utilized 43 of the 44 datasets to construct the regression equations. The remaining dataset was then utilized to estimate the mFIM $\hat{F}$. To evaluate the estimation accuracy, we calculated the MAE between the estimated mFIM $\hat{F}$ and $F_{\text {true }}$ assessed by the nurses using Eq. (5).

$$
M A E=\frac{1}{44} \sum_{i=1}^{44}\left|\hat{F}_{i}-F_{\text {true }}\right|
$$

To evaluate whether independently utilizing each axis or combining all axes for each bodily movement is optimal, eight $M A E$ were calculated in the case of combining all three axes for each target bodily movement. 
Table 1: Parameters for the model based on rising from a bed for a healthy person and squared error

\begin{tabular}{c|r|c|c|c|c|c}
\hline \multirow{2}{*}{ Axis } & \multicolumn{5}{|c|}{ Parameters in Eq. (1) } & \multirow{2}{*}{$\begin{array}{c}\text { Squared } \\
\text { error }\end{array}$} \\
\cline { 2 - 7 } & \multicolumn{1}{|c|}{$A$} & $\gamma$ & $\alpha$ & $\beta$ & $T$ & \\
\hline \hline $\mathrm{x}$ & 66.03 & 0.03 & 0.05 & 1.64 & 2.90 & 84.55 \\
\hline $\mathrm{y}$ & -553.34 & 0.06 & 0.08 & $9.45 \times 10^{-7}$ & 2.90 & 34.40 \\
\hline $\mathrm{z}$ & 103.76 & 0.04 & 0.09 & $1.63 \times 10^{-7}$ & 2.75 & 132.50 \\
\hline
\end{tabular}

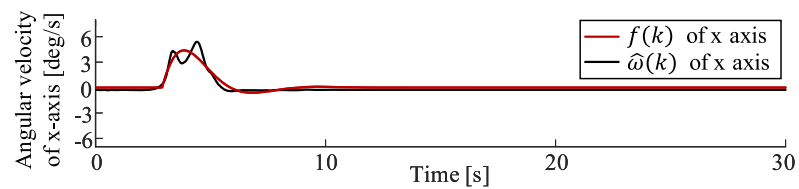

(a) $\mathrm{x}$ axis

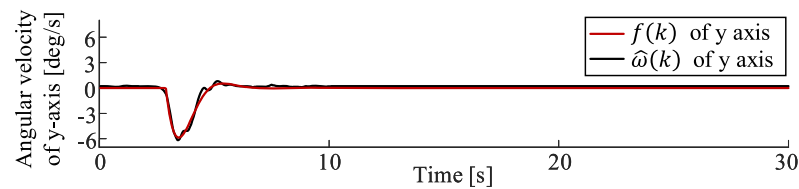

(b) y axis

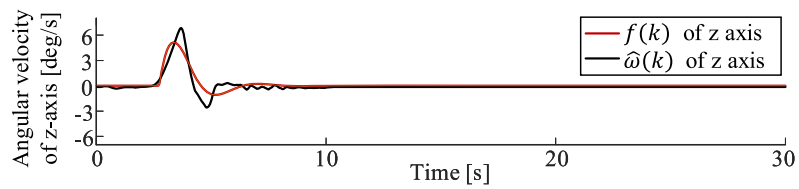

(c) $\mathrm{z}$ axis

Figure 4: Output of the model $f(k)$ and angular velocity, $\hat{\omega}(k)$ after preprocessing of each axis based on rising from a bed for a healthy person $\left(F_{\text {true }}=91\right)$

\section{EXPERIMENTAL RESULTS}

\subsection{Results of feature extraction}

In this section, we focus on the parameters $A, \gamma, \alpha, \beta$, and $T$ in Eq. (1), which were determined using curve fitting. Additionally, the outputs of the model $f(k)$ calculated by inputting the determined parameters into Eq. (1) and the angular velocities $\hat{\omega}(k)$ after preprocessing for each axis are presented.

1) Rising from a bed

Table 1 lists the five parameters determined for Eq. (1) and the squared error between $f(k)$ and $\hat{\omega}(k)$ based on rising from a bed for a healthy individual with an mFIM of 91. Additionally, the outputs of the model $f(k)$ were calculated by inputting the parameters in Table 1 into Eq. (1). The angular velocities $\hat{\omega}(k)$ after preprocessing are presented in Fig. 4. $f(k)$ is represented by the red line and $\hat{\omega}(k)$ is represented by the black line. As shown in Fig. 4, the angular velocities $\hat{\omega}(k)$ for rising from a bed are very simple time series data for each axis with only one large crest or trough. Additionally, as shown in Fig. 4, the time required for the movement to be completed was short. The movement itself was completed in
Table 2: Parameters for the model based on rising from a bed for a stroke patient and squared error

\begin{tabular}{|c|c|c|c|c|c|c|}
\hline \multirow{2}{*}{ Axis } & \multicolumn{5}{|c|}{ Parameters in Eq. (1) } & \multirow{2}{*}{$\begin{array}{l}\text { Squared } \\
\text { error }\end{array}$} \\
\hline & $A$ & $\gamma$ & $\alpha$ & $\beta$ & $T$ & \\
\hline $\mathrm{x}$ & 11.93 & 0.02 & 0.15 & $7.64 \times 10^{-7}$ & 3.65 & 382.44 \\
\hline $\mathrm{y}$ & -5.98 & 0.01 & 0.08 & 2.07 & 1.60 & 170.08 \\
\hline $\mathrm{z}$ & 1.56 & 0.01 & 0.05 & 1.04 & 0.60 & 512.43 \\
\hline
\end{tabular}

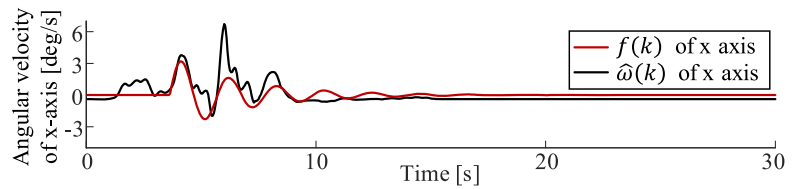

(a) $\mathrm{x}$ axis

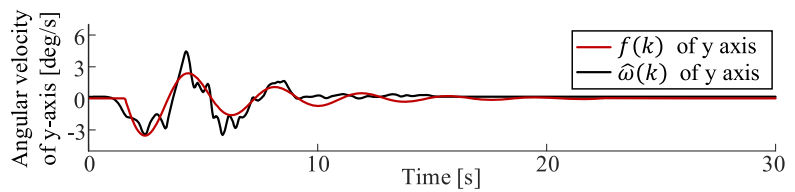

(b) y axis

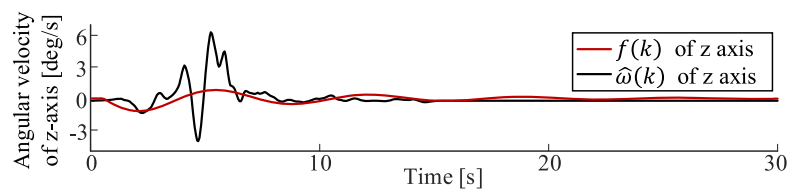

(c) $\mathrm{z}$ axis

Figure 5: Output of the model $f(k)$ and angular velocity, $\hat{\omega}(k)$ after preprocessing of each axis based on rising from a bed for a stroke patient $\left(F_{\text {true }}=52\right)$

approximately seven seconds. Therefore, because curve fitting performed well based on the simple time series of angular velocities $\hat{\omega}(k)$, each squared error value is low and the squared error for the $y$ axis is the lowest among the three axes at 34.40 .

The five parameters in Eq. (1) and the squared error based on rising from a bed for a stroke patient with an mFIM of 52 are presented in Table 2. The angular velocities $\mathrm{f}(\mathrm{k})$ corresponding to the parameters and $\hat{\omega}(k)$ are presented in Fig. 5. One can see that compared to the time series of angular velocities in Fig. 4, the angular velocities for the patient are more complex and it took more time for the movement to be completed. Therefore, the squared error for each axis is higher because curve fitting less precise. Additionally, even though the angular velocities of each axis were extracted from one movement, the dead time $T$ for the time series of each axis was different. The parameter $\gamma$ extracted from $\hat{\omega}(k)$ for the patient was lower than that for the healthy individual, the patient's movement converged more slowly.

For rising from a bed, we determined that the squared error between the angular velocity $\hat{\omega}(k)$ of the y axis and 
Table 3: Parameters for the model based on STS movement for the healthy individual and squared error

\begin{tabular}{c|r|c|c|c|c|c}
\hline \multirow{2}{*}{ Axis } & \multicolumn{5}{|c|}{ Parameters in Eq. (1) } & \multirow{2}{*}{$\begin{array}{c}\text { Squared } \\
\text { error }\end{array}$} \\
\cline { 2 - 7 } & \multicolumn{1}{|c|}{$A$} & $\gamma$ & $\alpha$ & $\beta$ & $T$ & \\
\hline \hline $\mathrm{x}$ & 147.17 & 0.04 & 0.09 & $4.66 \times 10^{-8}$ & 3.44 & 174.74 \\
\hline $\mathrm{y}$ & 246.81 & 0.04 & 0.09 & $3.55 \times 10^{-7}$ & 3.80 & 174.08 \\
\hline $\mathrm{z}$ & 72.01 & 0.04 & 0.14 & $5.79 \times 10^{-7}$ & 3.25 & 116.43 \\
\hline
\end{tabular}

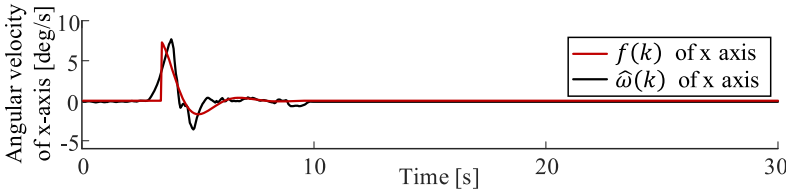

(a) $\mathrm{x}$ axis

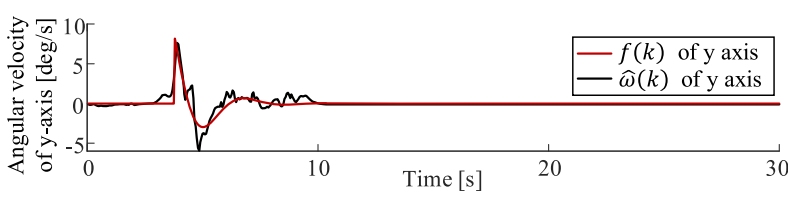

(b) y axis

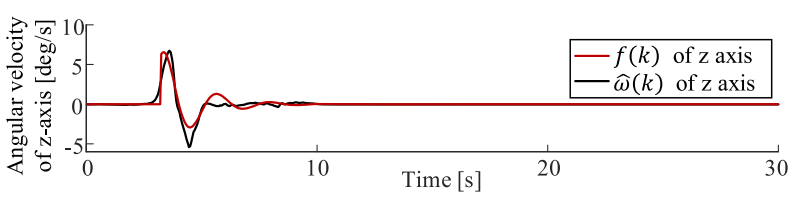

(c) $\mathrm{z}$ axis

Figure 6: Output of the model $f(k)$ and angular velocity $\hat{\omega}(k)$ after preprocessing for each axis based on STS movement for a healthy person $\left(F_{\text {true }}=91\right)$

the output of the model $f(k)$ for the $\mathrm{y}$ axis was the lowest among the three axes, regardless of the participant. Additionally, because each damping coefficient $\gamma$ for each participant was similar, each time series converged at a similar time, as shown in Fig. 5.

2) For STS movement

In the same way as for rising from the bed, the five parameters determined in Eq. (1) and the squared error based on the STS movement for a healthy person are shown in Table 3. This is the same participant whose information obtained from rising from a bed was presented in Table 1 and Fig. 4. Figure 6 presents the output of the model $f(k)$ and $\hat{\omega}(k)$ based on the STS movement for a healthy person. Similar to the time series for the angular velocities $\hat{\omega}(k)$ based on rising from a bed, those of the angular velocities $\hat{\omega}(k)$ based on STS movement are simple. However, in Fig. 6, one can see that the angular velocities $\hat{\omega}(k)$ based on STS movement contain more small oscillations than the $\hat{\omega}(k)$ based on rising from a bed. Additionally, as shown in Fig. 6, the waveforms of $\hat{\omega}(k)$ for each axis are similar. Although the squared errors for the $\mathrm{x}$ and $\mathrm{y}$ axes in Table 3 are greater than those in Table 1, one can see that curve
Table 4: Parameters for the model based on STS movement for the stroke patient and squared error

\begin{tabular}{c|c|c|r|r|c|c}
\hline \multirow{2}{*}{ Axis } & \multicolumn{5}{|c|}{ Parameters in Eq. (1) } & \multirow{2}{*}{$\begin{array}{c}\text { Squared } \\
\text { error }\end{array}$} \\
\cline { 2 - 6 } & \multicolumn{1}{|c|}{$A$} & $\gamma$ & \multicolumn{1}{c}{$\alpha$} & $\beta$ & \multicolumn{1}{c}{$T$} & \\
\hline \hline $\mathrm{x}$ & -2.35 & 0.01 & 0.09 & 0.15 & 1.50 & 491.75 \\
\hline $\mathrm{y}$ & -97.14 & 0.01 & 0.003 & 7.38 & 5.40 & 193.50 \\
\hline $\mathrm{z}$ & -5.90 & 0.01 & 0.06 & 8.26 & 3.45 & 244.19 \\
\hline
\end{tabular}

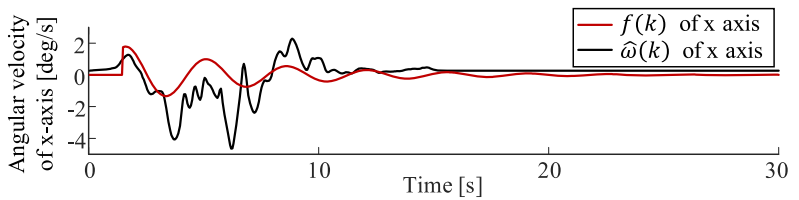

(a) $\mathrm{x}$ axis

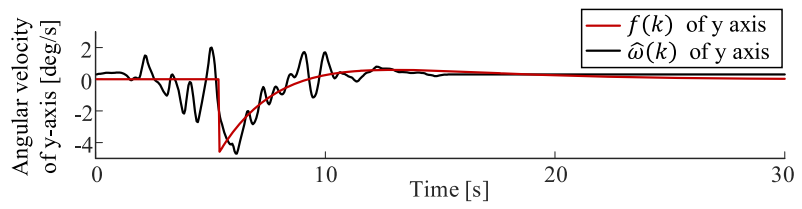

(b) y axis

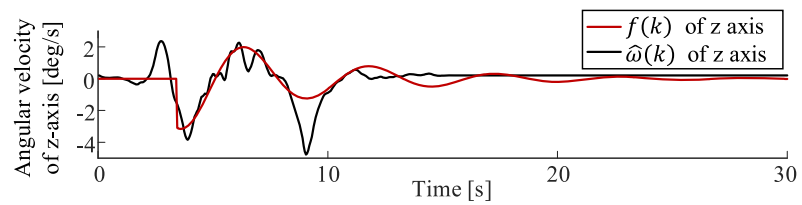

(c) $\mathrm{z}$ axis

Figure 7: Output of the model $f(k)$ and angular velocity $\hat{\omega}(k)$ after preprocessing for each axis based on STS movement for a stroke patient $\left(F_{\text {true }}=52\right)$

fitting still worked well overall. The squared error for the $\mathrm{z}$ axis is lower than that in Table 1 and curve fitting also worked well. Additionally, the parameter $\gamma$ extracted from $\hat{\omega}(k)$ is the same for all axes. Therefore, we can assume that the speed of convergence is similar for all axes.

Table 4 and Fig. 7 present the parameters in Eq. (1), as well as $f(k)$ and $\hat{\omega}(k)$, based on STS movement for the same stroke patient participant whose information was presented in Table 2 and Fig. 5. As shown in Fig. 7, for the time series of $\hat{\omega}(k)$, particularly the $\hat{\omega}(k)$ of the $\mathrm{x}$ and $\mathrm{y}$ axes, relatively large oscillations occur from 1 to $12 \mathrm{~s}$. Therefore, curve fitting did not work well and the squared error is large. Additionally, similar to the dead times $T$ in Table 2, those shown in Table 4 are different for each axis despite all coming from time series for the same movement.

For STS movement, we found that the squared error between the angular velocity $\hat{\omega}(k)$ along the $\mathrm{z}$ axis and the output of the model $f(k)$ along the $\mathrm{z}$ axis was the lowest among the three axes, regardless of the participant. Additionally, each parameter $\gamma$ for each participant was the same. 
Table 5: MAEs calculated based on the mFIM $\hat{F}$ estimated by utilizing features obtained from three axes for each movement

\begin{tabular}{c|c|c|c}
\hline & \multirow{2}{*}{ Axis } & \multicolumn{2}{|c}{ Target bodily movement } \\
\cline { 3 - 4 } & & Rising from a bed & STS movement \\
\hline \hline \multirow{3}{*}{ MAE } & $\mathrm{x}$ & $12.44 \pm 15.25$ & $13.11 \pm 16.07$ \\
\cline { 2 - 4 }$($ Mean \pm Std) & $\mathrm{y}$ & $9.53 \pm 12.59$ & $12.12 \pm 15.16$ \\
\cline { 2 - 4 } & $\mathrm{z}$ & $12.79 \pm 15.41$ & $10.23 \pm 12.86$ \\
\cline { 2 - 4 } & $\mathrm{x}, \mathrm{y}, \mathrm{z}$ & $10.86 \pm 13.96$ & $11.04 \pm 13.65$ \\
\hline
\end{tabular}

5.2 MAEs in the case of applying each axis or combining all axes for each movement

Table 5 lists the MAEs calculated utilizing the angular velocities of each axis for each movement and those of all axes for each movement. Among the eight types of $M A E$ s, the $M A E$ based on the angular velocities $\omega(k)$ along the $\mathrm{y}$ axis for rising from a bed is the lowest $(M A E=9.53)$, whereas that based on the angular velocities $\omega(k)$ along the $y$ axis for STS movement are the highest $(M A E=13.11)$. Considering only the MAE of the STS movement, the smallest error resulted from the angular velocities of $\mathrm{z}$ axis. When the angular velocities $\omega(k)$ of all axes were used, the MAE based on each movement was the second lowest for each movement. We believe that the estimation accuracy of mFIM decreased compared with using only a single axis because the two axes with low estimation accuracy strongly affect the axis with the highest estimation accuracy when all axes are used.

\section{DISCUSSION}

In our experiments, we found that the angular velocities $\omega(k)$ along the $y$-axis in the direction of the spinal cord when rising from a bed yielded the best results $(M A E=$ 9.53). The result is equivalent to an average error of 0.73 $(=9.53 / 13)$ for each of the 13 items of the mFIM. Hence, because the resolution of mFIM scored by the nurse is one, a $M A E$ of 9.53 , with the average error for each item being less than minimum resolution, is considered as high accuracy; and we confirmed that the proposed system could estimate current mFIM with an accuracy equivalent to an error of less than one per item by measuring the rising from a bed movement once in a home environment. Table 6 lists all 44 datasets of the $F_{\text {true }}$ values assessed by the experienced nurses, the estimated mFIM $\hat{F}$, and the error defined as $F_{\text {true }}-\hat{F}$ based on the angular velocities $\omega(k)$ along the $y$-axis when rising from a bed. As shown in Table 6, among the 44 datasets, 22 datasets had estimated $\hat{F}$ values larger than $F_{\text {true }}$. In particular, $\hat{F}$ tended to be highly estimated for participants with $F_{\text {true }}$ values in the upper $60 \mathrm{~s}$ to the $90 \mathrm{~s}$. In contrast, the other 20 datasets, with $F_{\text {true }}$ values from the $20 \mathrm{~s}$ to the lower $70 \mathrm{~s}$, yielded
Table 6: The $F_{\text {true }}$ assessed by the nurses, estimated mFIM $\hat{F}$, and error based on the angular velocities $\omega(k)$ along the y axis for rising from a bed

\begin{tabular}{|c|c|c|}
\hline$F_{\text {true }}$ & $\hat{F}$ & Error \\
\hline 91 & 68.77 & 22.23 \\
\hline 91 & 91.00 & 0.00 \\
\hline 91 & 87.24 & 3.76 \\
\hline 91 & 62.19 & 28.81 \\
\hline 91 & 87.06 & 3.94 \\
\hline 91 & 83.94 & 7.06 \\
\hline 91 & 86.99 & 4.01 \\
\hline 91 & 87.23 & 3.77 \\
\hline 91 & 86.23 & 4.77 \\
\hline 91 & 76.00 & 15.00 \\
\hline 91 & 80.45 & 10.55 \\
\hline 91 & 91.00 & 0.00 \\
\hline 91 & 75.75 & 15.25 \\
\hline 89 & 65.61 & 23.39 \\
\hline 83 & 72.09 & 10.91 \\
\hline 80 & 63.25 & 16.75 \\
\hline 80 & 66.84 & 13.16 \\
\hline 75 & 64.83 & 10.17 \\
\hline 74 & 67.91 & 6.09 \\
\hline 73 & 80.34 & $\begin{array}{l}-7.34 \\
\end{array}$ \\
\hline 73 & 79.92 & -6.92 \\
\hline 71 & 71.58 & -0.58 \\
\hline 70 & 64.44 & 5.56 \\
\hline 69 & 64.15 & 4.85 \\
\hline 68 & 64.14 & 3.86 \\
\hline 67 & 75.26 & -8.26 \\
\hline 67 & 64.60 & 2.40 \\
\hline 65 & 64.73 & 0.27 \\
\hline 63 & 65.58 & -2.58 \\
\hline 63 & 64.95 & -1.95 \\
\hline 63 & 67.74 & -4.74 \\
\hline 62 & 64.75 & -2.75 \\
\hline 61 & 65.70 & -4.70 \\
\hline 61 & 67.74 & -6.74 \\
\hline 60 & 65.11 & -5.11 \\
\hline 59 & 71.39 & -12.39 \\
\hline 59 & 65.16 & -6.16 \\
\hline 54 & 66.98 & -12.98 \\
\hline 54 & 70.41 & -16.41 \\
\hline 52 & 66.37 & -14.37 \\
\hline 50 & 65.67 & -15.67 \\
\hline 50 & 66.35 & -16.35 \\
\hline 48 & 66.27 & -18.27 \\
\hline 29 & 67.35 & -38.35 \\
\hline
\end{tabular}

estimated $\hat{F}$ values smaller than $F_{\text {true }}$. The average error of the 22 datasets in which the mFIM was estimated to be larger than the true value was 9.84 , and that of the 20 datasets in which the mFIM was estimated to be smaller than the true value was -10.13 .

In addition, we found that there were two issues with the trunk control model to be addressed to improve the overall estimation accuracy. First, when the error was larger, as shown in Figs. 5 and 7, the time series of the angular velocities exhibited small oscillations. Second, the time series of the angular velocity $\hat{\omega}(k)$ along the $\mathrm{z}$-axis after preprocessing in Fig. 7 began to oscillate again after converging. Therefore, in the future, to reduce the error 
between the estimated mFIMs $\hat{F}$ and $F_{\text {true }}$ assessed by the highly trained nurses, which resulted from the small oscillations and secondary oscillations that were not taken into account in the trunk control model, we plan to update the trunk control model to consider these aspects. If these two issues can be addressed, we expect that this system will estimate the mFIM more precisely because the curve fitting will perform better, and the corresponding parameter values will be determined more accurately.

\section{CONCLUSION}

In this paper, we proposed a system to aid stroke patients and caregivers in easily estimating mFIMs by performing a specific bodily movement only once in a home environment. We considered two types of target bodily movements as candidate movements for the specific bodily movement, namely rising from a bed and the STS movement. Healthy people can complete these movements smoothly and quickly, whereas stroke patients require more time to complete these movements based on degraded trunk function. Therefore, we constructed a model considering the differences in convergence speed between the target bodily movements of healthy individuals and those of stroke patients, and verified whether rising from a bed or the sit-to-stand movement is the optimal specific bodily movement. We estimated mFIMs by performing Gaussian process regression analysis with the parameters utilized in the constructed model as explanatory variables and the mFIMs assessed by highly trained nurses as objective variables. The parameters were extracted by curve fitting to match the angular velocities $\hat{\omega}(k)$ after denoising and normalization. After calculating the $M A E \mathrm{~s}$ in the cases of applying each axis or combining all axes for each movement, we determined that the $M A E$ based on the angular velocities $\omega(k)$ along the $\mathrm{y}$-axis (in the spine direction) when rising from a bed was the lowest at 9.53 .

\section{REFERENCES}

1. Statistics Bureau of Japan; 2020 statistical handbook of Japan, 2020.

2. Ministry of Health, Labor, and Welfare; 2014 summary of patient surveys, 2014.

3. Ministry of Health, Labor, and Welfare; Comprehensive survey of living conditions, 2016.

4. Heinemann, A. W., Linacre, J. M., Wright, B. D., Hamilton, B. B., and Granger, C.; Relationships between impairment and physical disability as measured by the functional independence measure,
Archives of Physical Medicine and Rehabilitation, 74(6), pp.566-573, 1993.

5. Linacre, J. M., Heinemann, A. W., Wright, B. D., Granger, C. V., and Hamilton, B. B.; The structure and stability of the functional independence measure, Archives of Physical Medicine and Rehabilitation, 75(2), pp.127-132, 1994.

6. Ottenbacher, K. J., Hsu, Y., Granger, C. V., and Fiedler, R. C.; The reliability of the functional independence measure: A quantitative review, Archives of Physical Medicine and Rehabilitation, 77(12), pp.1226-1232, 1996.

7. Brock, K. A., Goldie, P. A., and Greenwood, K. M.; Evaluating the effectiveness of stroke rehabilitation: Choosing a discriminative measure, Archives of Physical Medicine and Rehabilitation, 83(1), pp.92-99, 2002.

8. Wada, Y., Sonoda, S., Okamoto, S., Watanabe, M., Okazaki, H., and Okuyama, Y.; Comparison of prediction accuracy of the total score of FIM motor items at discharge in post-stroke patients in a Kaifukuki rehabilitation ward, Japanese Journal of Comprehensive Rehabilitation Science, 10, pp.71-76, 2019.

9. Hirota, T., Hamada, Y., Kaburagi, T., and Kurihara, Y.: Functional independence measure motor score estimation method considering instability of movement, Proceedings of the 7th International Symposium on Affective Science and Engineering, 8A-02, 2021.

10. Verheyden, G., Vereeck, L., Truijen, S., Troch, M., Herregodts, I., Lafosse, C., Nieuwboer, A., and Weerdt, W. D.; Trunk performance after stroke and the relationship with balance, gait, and functional ability, Clinical Rehabilitation, 20(5), pp.451-458, 2006.

11. Janssen, W.G. M., Bussmann, H.B. J., and Stam, H. J.; Determinants of the Sit-to-stand movement: A review, Physical Therapy, 82(9), pp.866-879, 2002.

12. Riley, P. O., Krebs, D. E., and Popat, R. A.; Biomechanical analysis of failed sit-to-stand, IEEE Transactions on Rehabilitation Engineering, 5(4), pp.353-359, 1997.

13. Lara-Barrios, C. M., Blanco-Ortega, A., AbúndezPliego, A., and Colín-Ocampo, J.; Sit-to-stand simulation for torque estimation on lower limb joints, Proceedings of the 2015 International Conference on Mechatronics, Electronics and Automotive Engineering (ICMEAE), pp.116-121, 2015.

14. Baer, G. D., and Ashburn, A. M.; Trunk movements in older subjects during sit-to-stand, Archives of Physical Medicine and Rehabilitation, 76(9), pp.844849, 1995. 


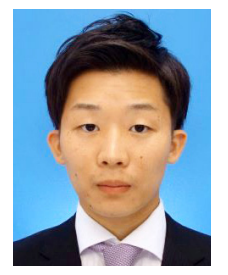

Taku HIROTA (Non-member)

He received the B.E. degree from Aoyama Gakuin University, Japan, in 2020, where he is currently pursuing the degree with the Graduate School. His research interests include system engineering, machine learning, and biosignal measurement.

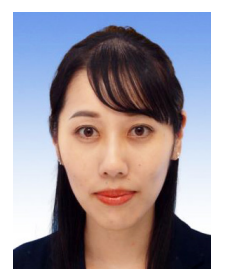

\section{Yuri HAMADA (Member)}

She received her B.S., M.S., and Ph.D. degrees in engineering from Chuo University, Japan, in 2008, 2010, and 2017, respectively. From 2018 to 2020, she served as an Assistant Professor at Chuo University. Since 2020, she has been working as an Assistant Professor in the Industrial and Systems Engineering department at the Aoyama Gakuin University. Her research interests include modeling of communication processes and analysis of decision-making processes. She is a member of the Japan Society of Kansei Engineering, the Japanese Cognitive Science Society, and IEEE.

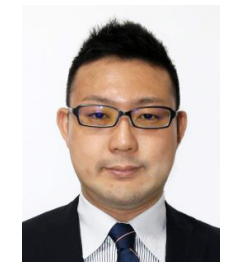

Takashi KABURAGI (Non-member)

He received his B.E., M.E., and Ph.D. degrees from Waseda University in 2003, 2005, and 2009, respectively. $\mathrm{He}$ is currently an Instructor at the College of Liberal Arts, International Christian University, where he has been a faculty member since 2019. His research interests include machine learning and time series data analysis.

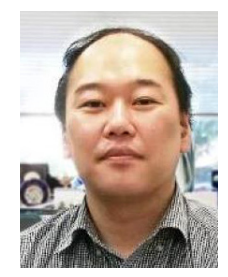

\section{Yosuke KURIHARA (Non-member)}

He received his M.E. and Ph.D. degrees from Hosei University, Tokyo, in 2003 and 2009, respectively. He joined Hitachi Software Engineering, Ltd., in 2003. From 2009 to 2012, he served as an Assistant Professor at Seikei University. From 2013 to 2018, he was an Associate Professor at Aoyama Gakuin University; and from 2019, he has been serving as a Professor. He is the author of five books, more than 80 journal papers, more than 100 international conference proceedings; and holds four patents. His research interests include system engineering, sensing methods, biosensing, and system information engineering. He is a member of the Japanese Society for Medical and Biological Engineering, the Society of Instrument and Control Engineers, the Electrical Engineers of Japan, the Japan Society for Fuzzy Theory and Intelligent Informatics, and the IEEE. 